\title{
Hydrodynamic drag and velocity of micro-bubbles in dilute paper machine suspensions
}

\author{
A. Haapala ${ }^{1}$, M. Honkanen ${ }^{2}$, H. Liimatainen ${ }^{1}$, \\ T. Stoor ${ }^{1} \&$ J. Niinimäki ${ }^{1}$ \\ ${ }^{1}$ University of Oulu, Fibre and Particle Engineering Laboratory, Finland \\ ${ }^{2}$ Tampere University of Technology, Energy and Process Engineering, \\ Finland
}

\begin{abstract}
This paper studies hydrodynamic drag forces acting on freely rising microbubbles in dilute paper machine suspensions under turbulent flow conditions. Dissolved, colloidal and numerous solid materials i.e. process chemicals, wood extractives, fillers and wood fibre fractions present in these suspensions disturb the rise of micro-bubbles increasing their drag. The aim of this study is to characterise the terminal velocities and drag coefficients of the bubbles as a function of their Reynolds number in several paper machine circulation waters, i.e. white waters, and in some model suspensions. Characterisation is performed experimentally with a high-speed CMOS camera and a submersed back-light illumination in a pressurised bubble column.

Image sequences of bubbly flow are analysed with automatic image processing algorithms that measure not only the bubble size and velocity, but also the velocity of the fluid surrounding bubbles, revealing the initial slip velocity of each bubble. Bubbles are tracked in time to provide time series data for every bubble that passes the focal plane of the imaging system. Results show how some suspension properties - concentration, apparent viscosity and surface tension - affect the motion of micro-bubbles. Results also show the changes in micro-bubble formation with pressure drop and differences of bubble size distributions in a variety of suspensions and solutions. Finally, a mathematical model describing the bubble rise velocities and drag coefficients with respect to the bubble Reynolds number is developed for the investigated white waters.
\end{abstract}

Keywords: bubble motion, drag coefficient, drag force, white water, papermaking, high-speed imaging, high-speed imaging, bubble sizing. 


\section{Introduction}

Bubbly gasses and their interaction with other substances in pulp suspensions may cause substantial problems in papermaking processes. Most of them relate to stock filtration, dewatering, sheet formation or pumping but also process measurements. For example, consistency can be affected as recently presented by Stoor [1] and Helle et al [2]. Deaeration in papermaking usually takes place in a pressurised tank or a special gas removing pump, while passive methods rely solely on bubble rise and evacuation from suspension. Today, different solutions for gas removal are developed using mathematical models and computational fluid dynamics (CFD) tools that describe the phenomenon of bubble motion. These models in turn require experimental knowledge on the kinetics and hydrodynamics behind the free gas bubble rise in process suspensions.

The rise of free gas bubbles in papermaking suspensions is hindered by apparent viscosity and solids, while surface tension of the liquid phase affects the size distribution of formed bubbles [3-8]. This is also typical for fibre-free filtration waters drained from sheet former, commonly referred as white waters. Suspended and dissolved solids are the main components contributing to bubble drag in white waters i.e. fibre fines fractions and inorganic particles such as clay fillers $[7,8]$. Their composition and overall chemical state varies from one paper machine to another, whilst process waters slowly accumulate dissolved and colloidal materials from raw materials, process chemicals and fresh water. While the effect of surfactants on the drag coefficient of rising bubbles was shown previously in the work of Okazaki [6] in 1962, so far research on experimental values for bubble drag and rise velocity in complex papermaking white waters has not been published.

Image-based measurements have become a powerful tool to determine the gas bubble size, velocity and the velocity of suspension surrounding the gas bubble $[8,9]$. Robust image analysis algorithms $[10,11]$ can automatically recognise individual, in-focus bubbles from image sequences of complex multiphase suspensions, where bubbles commonly overlap. Optical measurements are, however, limited to dilute multiphase flows, because they need undisturbed optical access to the measurement volume. Dispersed phase particles and bubbles in these suspensions scatter the incident light and damp the light intensity, which restricts the penetration of light through the suspension. In addition, they scatter light on the optical path between the illumination source and camera causing image distortions. Opaque multiphase suspensions, such as white waters, are often testing the limits of digital imaging methods. However, an underwater measurement probe [13] or illumination also provides a way to visualise these opaque multiphase suspensions inside large vessels. In this study, the bubbly multiphase flows are visualised close to the column wall with submerged backlight illumination optics.

The aim of the study is to investigate the effect of white water properties on size distribution and motion of micro-bubbles in white waters in order to produce consistent experimental data for the validation of CFD models that describe the deaeration phenomenon of these suspensions. Micro-bubbles are generated in 
characterised white waters and model waters of varying consistencies, apparent viscosities and surface tension by dissolving air under pressure and causing a sudden pressure drop. Generated micro-bubbles are then visualised using a highspeed CMOS camera and submerged diode laser back-light. Bubble size distributions, rise velocities of bubbles and bubble drag coefficients $\left(C_{D}\right)$ are defined for each white water as a function of bubble Reynolds number.

\section{Materials and methods}

\subsection{Studied suspensions}

The examined white waters (WWs) originate from 8 paper machines that utilise various raw materials and produce a variety of paper grades: mechanical pulp to produce newsprint and magazine grades while eucalyptus, pine or similar kraft pulp to produce different fine paper grades. Thus fines and fillers content in white waters varied accordingly for each machine.

Analyses on waters were made according to the following standards and methods; pH (SFS 3021), conductivity (SFS-EN 27888) and dry matter content (SFS-EN 20638). Surface tension was measured with a Krüss K8600 (du Noüy's ring method) and apparent viscosities with a Haake 501 viscometer. In addition, apparent mean particle size of white water solids were determined using a multiwavelength particle size analyser (Beckman Coulter LS 13 320). Physicochemical properties of tested white waters are summarised in table 1.

Table 1: $\quad$ Properties of white waters.

\begin{tabular}{|c|c|c|c|c|c|c|c|c|}
\hline & WW 1 & WW 2 & WW 3 & WW 4 & WW 5 & WW 6 & WW 7 & WW 8 \\
\hline $\mathrm{pH}\left(20^{\circ} \mathrm{C}\right)$ & 7.9 & 4.0 & 6.9 & 7.7 & 7.7 & 7.7 & 7.7 & 7.6 \\
\hline Density $\left(40^{\circ} \mathrm{C}\right)[\mathrm{g} / \mathrm{L}]$ & 988 & 989 & 988 & 987 & 991 & 991 & 995 & 989 \\
\hline $\begin{array}{c}\text { Conductivity } \\
\left(20^{\circ} \mathrm{C}\right)[\mu \mathrm{S} / \mathrm{cm}]\end{array}$ & 1.5 & 2.0 & 1.0 & 0.9 & 1.0 & 0.9 & 1.1 & 1.4 \\
\hline $\begin{array}{l}\text { Dry Matter } \\
\text { Content [\%] }\end{array}$ & 0.58 & 0.49 & 0.50 & 0.25 & 0.22 & 0.22 & 0.25 & 0.21 \\
\hline $\begin{array}{c}\text { Surface tension } \\
\left(20^{\circ} \mathrm{C}\right)[\mathrm{mN} / \mathrm{m}]\end{array}$ & 53 & 51 & 48 & 59 & 58 & 53 & 66 & 61 \\
\hline $\begin{array}{l}\text { Apparent viscosity } \\
\left(40^{\circ} \mathrm{C}\right)[\mathrm{mPas}]\end{array}$ & 1.7 & 1.8 & 1.8 & 1.4 & 1.3 & 1.2 & 1.4 & 1.5 \\
\hline $\begin{array}{c}\text { Mean particle size, } \\
\text { (from }<90 \% \text { ) }[\mu \mathrm{m}]\end{array}$ & $7.9(20.7)$ & $10.0(26.3)$ & $6.5(20.8)$ & $7.3(23.6)$ & $7.4(20.0)$ & $8.3(26.3)$ & 10.4 (33.3) & $7.1(19.4)$ \\
\hline
\end{tabular}

In addition to process waters some measurements were made on model waters. Carboxymethyl cellulose (CMC) and n-butanol were added into deionised water in the absence and presence of dry refined pine kraft pulp fibres (average fibre length $1.52 \mathrm{~mm}$ ).

$\mathrm{CMC}$ was used to increase apparent viscosity, n-butanol to lower suspension surface tension and wood fibres to promote micro-bubble formation through nucleation and also to increase bubble drag through bubble-solid interactions in model waters. Micro-bubble motion in a variety of these suspensions was similarly measured in order to characterise the effects of fibre and fines content, suspension viscosity and surface tension to micro-bubble movement and size distribution. Properties of these model waters are presented in table 2. 
Table 2: $\quad$ Properties of model suspensions.

\begin{tabular}{|c|c|c|c|c|c|}
\hline & Water & CMC & CMC + fibre & n-but & n-but + fibre \\
\hline $\left.\mathbf{p H ~ ( 2 0}{ }^{\circ} \mathbf{C}\right)$ & 8.6 & - & - & - & - \\
\hline $\begin{array}{c}\text { Dry Matter } \\
\text { Content [\%] }\end{array}$ & 0.0 & 0.0 & 0.15 & 0.0 & 0.15 \\
\hline $\begin{array}{c}\text { Surface tension } \\
\left(\mathbf{2 0}{ }^{\circ} \mathbf{C}\right)[\mathrm{mN} / \mathrm{m}]\end{array}$ & 72 & 68 & 70 & 54 & 54 \\
\hline $\begin{array}{c}\text { Apparent viscosity } \\
\left(\mathbf{2 0}^{\circ} \mathbf{C}\right)[\mathrm{mPas}]\end{array}$ & 1.0 & 5.6 & 5.8 & 1.5 & 2.2 \\
\hline
\end{tabular}

\subsection{Measurement setup}

Experiments were carried out in a batch process, where 20 litres of suspension was pressurised inside a $1200 \mathrm{~mm}$ long cylindrical bubble column with a diameter of $167 \mathrm{~mm}$. A steady internal overpressure of 3 bars was maintained by feeding $25^{\circ} \mathrm{C}$ pressurised air through sintered porous media at 4 bars, allowing continuous air flow-through in column. The amount of dissolved oxygen in water increased until the suspension saturation level was reached. This was monitored with a dissolved oxygen analyser (Hach Orbisphere 3600).

After saturating the suspension, a pressure drop was created by opening a solenoid valve on top of the column. Images of generated micro-bubbles were gathered over a period of 30 seconds after the first micro-bubble appeared in the measurement volume. Illustration of the experimental setup is presented in fig. 1 . The column had a $300 \mathrm{~mm}$ high midsection of transparent polycarbonate piping through which imaging took place. The mill waters were tested at $40^{\circ} \mathrm{C}$ to achieve similar physical suspension characteristics that occur in real process environments, while tests on model suspension took place at $20^{\circ} \mathrm{C}$.

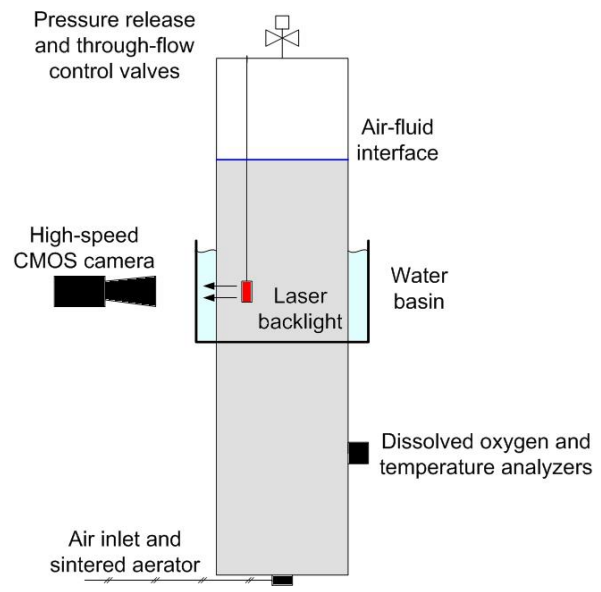

Figure 1: The test setup consisting of pressurised bubble column and camera. 


\subsection{Measurement technique}

A high-speed digital imaging setup was used to visualise the rise of microbubbles near the bubble column wall. Imaging was limited only to near wall regions, as contaminants blocked the view deeper within the suspension. The flow was illuminated with a submerged light diffuser connected to a pulsed diode laser (Cavilux Smart, $400 \mathrm{~W}, 690 \mathrm{~nm}$ ) with an optical fibre. Cavilux control unit synchronised the laser and the high-speed CMOS camera (PCO 1200hs) while the image acquisition was user controlled with a laptop computer.

As shown in fig. 1, a camera was placed outside the column opposite to the submerged light diffuser to provide shadow images of micro-bubbles in the flow between the light diffuser and the column wall. To eliminate the image distortions due to the curved column wall, an external cubical basin filled with water was placed around the bubble column. Camera image was geometrically calibrated with a measuring rod and a scaling of $18.6 \mu \mathrm{m}$ per image pixel was obtained. Micro-bubbles and their motion were then detected as shown in fig. 2.
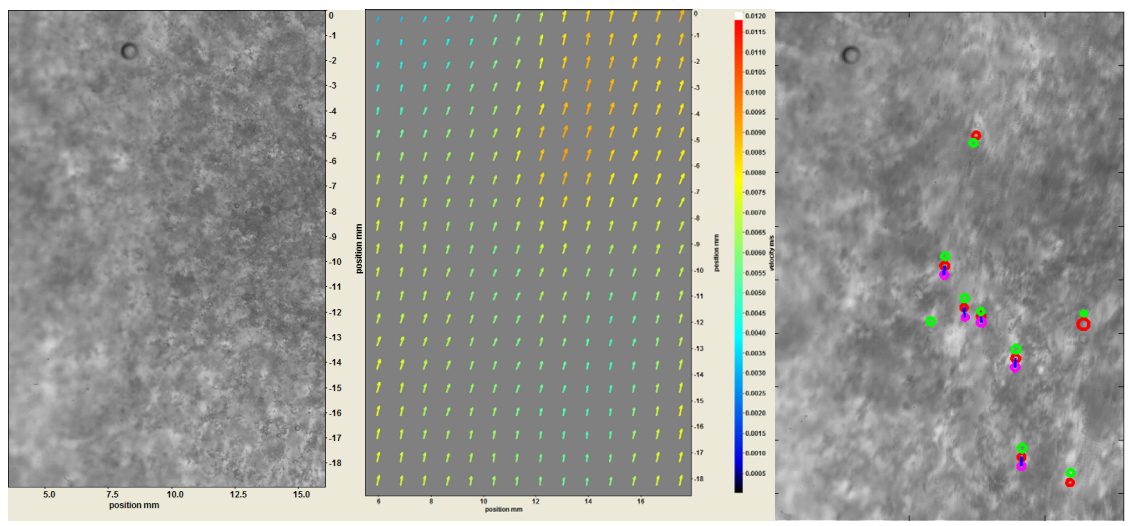

Figure 2: Original snapshot image of micro-bubbles in white water on left, the measured velocity vector field in the middle and a multi-frame image of detected micro-bubbles on right.

High-speed image sequences were analysed automatically with image analysis algorithms while a velocity vector field was provided by crosscorrelation of two consecutive images utilising the $32 \times 32$ pixel interrogation areas. Fast Fourier transform (FFT) and spatial vector validation were made with DaVis 7.2 software that is commonly utilised in particle image velocimetry (PIV) experiments.

Visual detection of micro-bubbles in the image was difficult even for a human eye. Micro-bubble recognition (human and automated) relies on the following assumptions: micro-bubbles produce dark, circular shadow images with a bright spot at the centre of the shadow and a sharp outline, whose curvature is nearly constant. We also assumed a size range from $50 \mu \mathrm{m}$ to $1 \mathrm{~mm}$ for micro-bubbles and that they are rising upwards, thus eliminating bubbles stuck on backlight or column walls, one of which is present up in the rightmost picture in fig. 2 . 


\subsection{Image analysis}

Automatic micro-bubble recognition algorithm included several phases that are briefly described in the following 5 step list:

1. Image equalisation: Image background was computed for each image with a low-frequency filter. Spatial image equalisation was obtained by dividing the image with the computed background image.

2. Detection of micro-bubble outlines: Local $(5 \times 5)$ standard deviation and a local kurtosis $\left(4^{\text {th }}\right.$ moment $)$ of greyscales emphasised the image areas of high greyscale variance. As local kurtosis on micro-bubble outline was greatly lower than that of curly fibre. Due to the presence of a smooth continuous change in the curvature of a bubble perimeter, the subtraction of a scaled local kurtosis image from the local standard deviation image returned an image emphasising only the outlines of micro-bubbles. Finally, a search for modified local maximum was carried out to recognise the outlines of sharp micro-bubble images.

3. Fitting circles on micro-bubble outlines: Micro-bubble was recognised by fitting a circle on its recognised outline. A fast and robust 3-point circle fit (modification of Kamgar-Parsi and Netanyahu [14]) was utilised.

4. Validation of circle fit: Fitted circles were validated based on size range, ratio of circle perimeter to recognised outline length and a ratio of circle area to area that overlaps with other circles, based on a criteria that the centre of the circle had to have higher original greyscale value than the inner side of the circle perimeter.

5. Lagrangian tracking of micro-bubbles in image sequence: After the recognition of micro-bubbles, three consecutive image frames were analysed to cluster images that belonged to the same micro-bubble. Thus each analysed micro-bubble had to be detected in at least three consecutive images. Only the rising micro-bubbles were of interest and thus the allowed velocity range was limited upwards. A constant rise velocity and size for each micro-bubble were assumed allowing a maximum of $20 \%$ variation in pseudo-distance s; defined as:

$$
s_{k, j}=\sqrt{\left(x_{i, e}(k)-x_{i}(j)\right)^{2}+\left(y_{i, e}(k)-y_{i}(j)\right)^{2}+\left(d_{i-1}(k)-d_{i}(j)\right)^{2}}
$$

where the bubble coordinates and size were predicted from the first two frames for the third image frame, and estimates $\left(\mathrm{x}_{\mathrm{i}, e,}, \mathrm{y}_{\mathrm{i}, \mathrm{e}}\right.$ and $\left.\mathrm{d}_{\mathrm{i}-1}\right)$ were compared to potential pair image values $\left(\mathrm{x}_{\mathrm{i}}, \mathrm{y}_{\mathrm{i}}\right.$ and $\left.\mathrm{d}_{\mathrm{i}}\right)$. The tracking method corresponded to a best-estimate method presented by Ouellette et al [15].

\subsection{Bubble size and velocity discretisation}

Digital imaging technique provided plenty of information on objects under study: object size, shape and concentration in the image. The relation of an object image size and real object size was straight-forward when the objects were spherical and the geometrical calibration between the image plane and the object plane was provided, as in the case of this study.

The relation between object concentration in image and in suspension can be obtained in dilute concentrations [16] and the size and shape of non-spherical objects can be statistically measured from a large set of images [12]. The gas 
bubbles in this application were spherical and their size range was narrow, so the computation of size distributions was provided directly from image analysis results. Size distributions are discretised into bubble size classes with equal width of $0.05 \mathrm{~mm}$ and size range from 0.1 to $1 \mathrm{~mm}$.

\subsection{Computation of the bubble drag co-efficient}

Bubble size, shape, rise velocity and the velocity of the surrounding fluid were measured with the imaging technique. Assuming a steady flow where only drag and buoyancy forces affect bubble motion, the bubble drag co-efficient was estimated. Suspensions were so dilute that the interactions of micro-bubbles could be neglected, but interactions between suspension contaminants and microbubbles clearly took place. These interactions were considered in the drag coefficient $\left(C_{D}\right)$ of a bubble. Thus, we obtained a simplified momentum equation that covers micro-bubble motion:

$$
\sum \vec{F}=\frac{1}{2} C_{D} \cdot \rho_{L} \cdot\left|\overrightarrow{U_{B}}-\overrightarrow{U_{L}}\right| \cdot\left(\overrightarrow{U_{B}}-\overrightarrow{U_{L}}\right) \cdot A_{B}+\left(\rho_{B}-\rho_{L}\right) \cdot V_{B} \cdot \vec{g}=0,
$$

where $A_{B}, V_{B}$ and $U_{B}$ are bubble's surface area, volume and rise velocity, $\rho$ is fluid density and $U_{L}$ is liquid velocity, obtained as the instantaneous mean liquid velocity in the whole measurement volume. In the case of spherical gas bubbles, momentum eq. (4) reduces to present $\mathrm{C}_{\mathrm{D}}$ in the following form:

$$
C_{D}=\frac{-\left(\rho_{B}-\rho_{L}\right)}{\rho_{L}} \cdot \frac{4}{3} \cdot \frac{d_{B} g}{\left(\overrightarrow{U_{B}}-\overrightarrow{U_{L}}\right)^{2}} \approx \frac{4}{3} \cdot \frac{d_{B} g}{\left(\overrightarrow{U_{B}}-\overrightarrow{U_{L}}\right)^{2}} .
$$

The drag co-efficient of a gas bubble rising in a stagnant pure liquid can be computed as a function of the bubble's Reynolds number. Depending on the bubble Reynolds number range used, $\mathrm{Re}_{\mathrm{B}}$ can be from either HadamardRybzynski (for $\mathrm{Re}_{\mathrm{B}} \leq 11$ ) [17] or Moore's solution (for $11<\mathrm{Re}_{\mathrm{B}} \leq 500$ ) [18]. However, Mei et al [19] obtained an empirical correlation that matches both correlations, valid for micro-bubble Reynolds numbers presented in this study:

$$
C_{D}=\frac{16}{\operatorname{Re}_{B}}\left\{1+\left[\frac{8}{\operatorname{Re}_{B}}+\frac{1}{2}\left(1+3.315 \operatorname{Re}_{B}{ }^{-1 / 2}\right)^{-1}\right]\right\} .
$$

To further quantify measured rise velocities, a comparative analysis was performed where the measured data was fitted to Mei's eqn (4), describing drag coefficients required to model the ascent of spherical bubbles in suspensions.

\section{Results and discussion}

Bubble dynamics in suspension flow differ from dynamics of a pure air/water suspension. During their rise, bubbles have to push solids away from their path. In addition, fibres in model suspensions dampen the flow velocity fluctuations and resist the flow from becoming turbulent, as reported in $[3,4,12]$. In process waters, bubbles were seen to align in swarms more strongly than in pure gasliquid suspension. The first bubble of a bubble swarm rises slower than the bubbles in its wake increasing the chances of bubble coalescence. On the other hand, surface active contaminants present in these suspensions induce bubble 
surface stabilisation, prevent bubble coalescence and lower bubble surface tension [6]. High-speed image sequences on model suspensions show that particles often attach on micro-bubbles and decrease their rise velocity, similar to phenomena seen in pulp suspensions [4]. Commonly, the buoyancy of a microbubble is not sufficient to detach the bubble from flocculated fines and thus some bubbles tend to remain trapped within suspension, making them naturally tedious to deaerate completely without any special process stages $[2,4,20]$.

\subsection{Micro-bubble size distribution in tested suspensions}

Distributions of micro-bubbles $\left(\mathrm{d}_{\mathrm{b}}<1.0 \mathrm{~mm}\right)$ after depressurising air-saturated suspensions from 3 bars to normal pressure are presented in fig. 3 . The bubbles generated in pure liquids are larger than bubbles in fibre suspensions. The presence of contaminants in every suspension shifts the distribution towards smaller bubble size. Fibre content appears to have even higher impact on mean bubble size than changes in surface tension and viscosity. Although increased fibre consistency is also noted to promote large bubble formation [3, 4], that trend is not visible here. Actually fewer micro-bubbles are generated in fibre suspensions than in pure liquids.
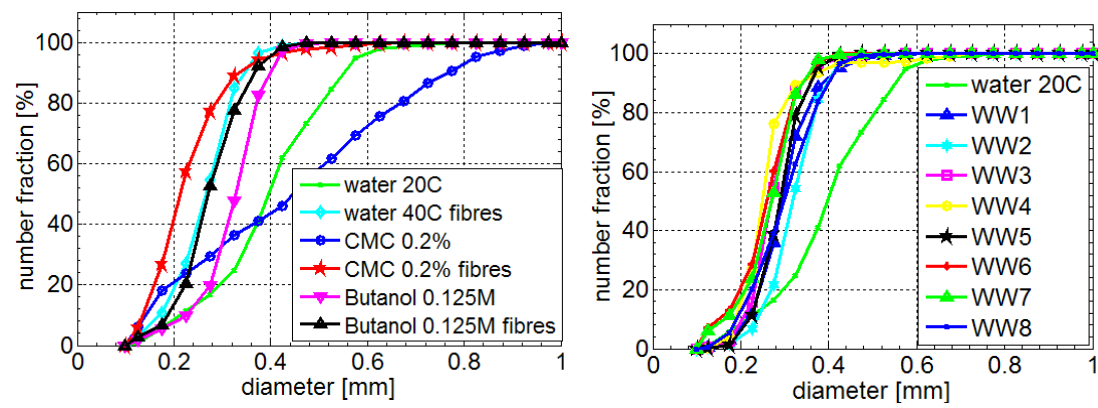

Figure 3: Micro-bubble size distribution on pressure drop for model suspensions and white waters.

Differences in bubble size distributions between white waters are small despite the differences in water properties. All white waters have sufficient solid contents to act as nucleation sites in the event of depressurisation and bubble formation, thus increasing the probability of small bubble formation. The differences in oxygen saturation levels on white waters are measured in few ppms and thus it seems that the chemical and physical properties of these white waters have little effect on the formed bubble size distribution. White waters produce micro-bubbles with similar size distribution to $0.125 \mathrm{M}$ n-butanol solution that has similar surface tension and apparent viscosity. However, the generated micro-bubble concentrations differ significantly.

\subsection{Bubble behaviour in model suspensions}

Fig. 4 shows sequences of three consecutive images overlaid on top of each other. The circles on top of the images present the detected in-focus bubbles and 
the blue arrows correspond to their velocities. Fig. 4 demonstrates how already the experimental images reveal the differences of suspensions. For example, CMC solution produces large bubbles that have higher tendency to attach on solid surfaces. The stagnant bubbles remain undetected and are not analysed.

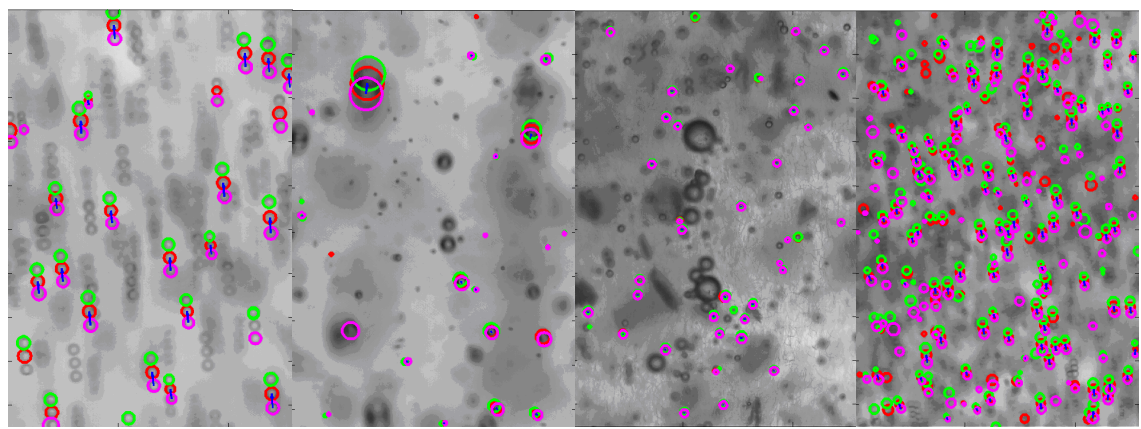

Figure 4: Micro-bubble analysis images on some measured cases: left, micro-bubbles in deionised water, $0.2 \% \mathrm{CMC}$ solution, solution of $0.2 \% \mathrm{CMC}$ and $0.15 \%$ fibres, and on right bubbles in $0.125 \mathrm{M} \mathrm{n}$ butanol.
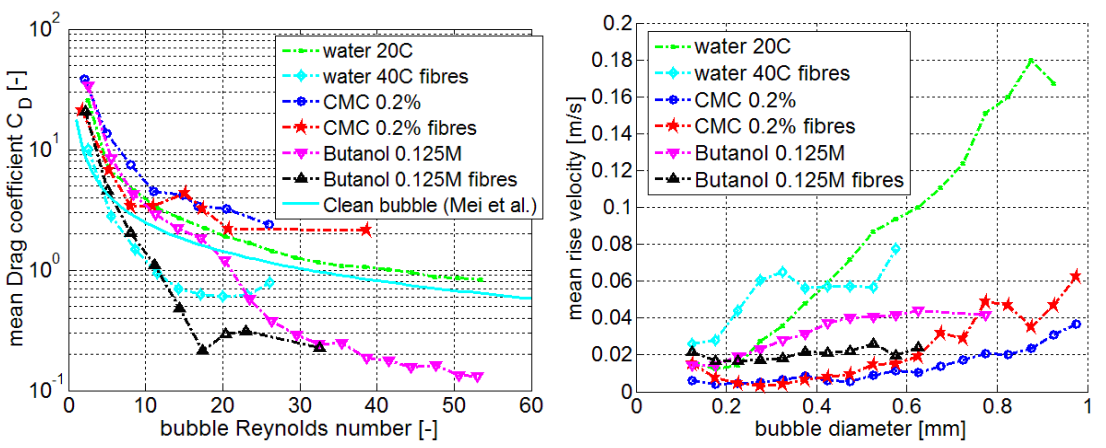

Figure 5: $\quad$ Bubble drag coefficients and mean bubble rise velocities according to micro-bubble Reynolds number and size for model suspensions.

Fig. 5 presents the measured drag coefficients $\left(C_{D}\right)$ with respect to bubble Reynolds number $\left(\mathrm{Re}_{\mathrm{B}}\right)$ and the measured rise velocities with respect to bubble diameter. As seen in tables 1 and 2, the model suspensions had lower solid contents than any process water. Suspensions with CMC had high viscosities and those with n-butanol were at equal level to mill waters both in terms of apparent viscosity and surface tension. As can be seen in Fig. 5 micro-bubble drag is elevated by suspension viscosity and lowered by surface tension - presence of fibres don't seem to have significant impact on bubble drag, likely due to channelling of rising bubbles to areas with lowest consistencies. 
The bubble rise velocity in water and liquid-solid suspensions with low solids consistencies can be reasonably estimated using the Mei's model. Measured $C_{D}$ differs somewhat from eqn (4) for bubbles with low $\mathrm{Re}_{\mathrm{B}}$, seemingly experiencing an exponential decline as $\mathrm{Re}_{\mathrm{B}}$ grows. Deviations from Mei's model seem to arise in suspensions of higher apparent viscosity, which was expected given that the model is based upon uncontaminated liquid medium. Presence of fibres appears less significant suggesting that apparent viscosity is the property most hindering micro-bubble rise in suspensions.

Similarly, bubbles in the most viscous suspensions had by far the slowest rise velocities within all size categories, as seen in fig. 5. While all model waters had reduced rise velocity in relation to pure water, viscous (over $5.5 \mathrm{mPas}$ ) CMCsolutions affected bubble motion most. Bubble rise in surfactant treated suspensions with viscosity around $2 \mathrm{mPas}$ was faster, but still far from reference waters. Fibres are supposed to hinder bubble rise mainly through collisions and attachment, even though these have a minimal affect bubbles can readily avoid entanglement and no web formation occurs in waters this diluted. Also fibres present in CMC-solutions appear insignificant in relation to viscous drag of the liquid phase.

\subsection{Bubble behaviour in white waters}

Small changes in physico-chemical properties of white waters made detailed analysis on drag or terminal velocity of bubbles problematic. In relation to deionised water, the drag of the smallest bubbles in papermaking suspensions was, however, clearly elevated, as seen in fig. 6 left. As in model waters, the most remarkable deviations from Mei's theory [19] occur for bubbles with the smallest $\mathrm{Re}_{\mathrm{B}}$ values. This is mainly attributed to surface contamination of bubbles that reduces surface tension and to elevate viscosity of the white water's liquid phase. The uniformity of drag coefficient development on suspensions allows generalisations to be made in modelling work for most typical mill white waters as bubble rise media. Drag coefficient of micro-bubbles is higher in white waters than in pure water when $\operatorname{Re}_{\mathrm{B}}<10$ and lower when $\mathrm{Re}_{\mathrm{B}}>10$.
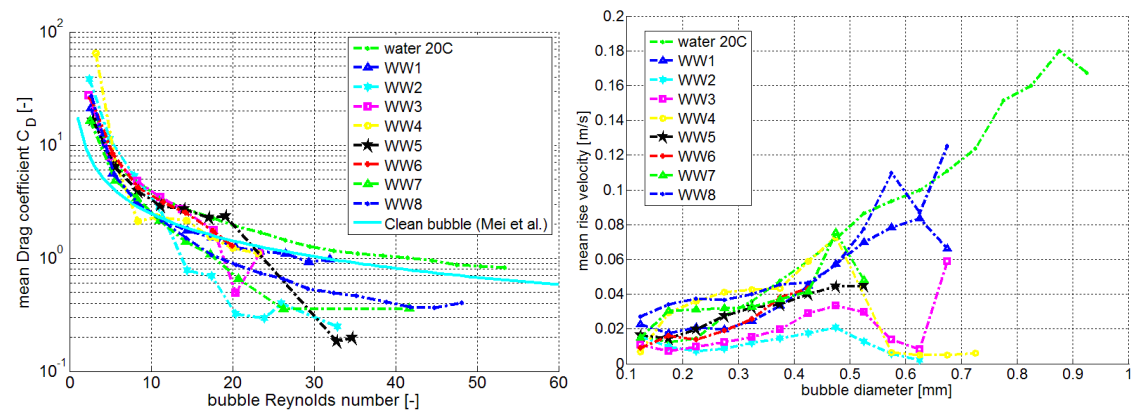

Figure 6: Bubble drag coefficients and mean bubble velocities according to the micro-bubble Reynolds number and size for white waters. 
The non-linearity of certain drag and micro-bubble velocity measurements can be attributed to a small population of detected bubbles in each size class and to the attachment of some, especially larger micro-bubbles on to pulp fragments. This was mostly seen on white waters 2,3 and 4 in fig. 6 . Based on experiences with different suspensions, we can conclude that the differences in solid contents and deviations in apparent viscosity or suspended particle sizes are so minor that they can be approximated with a single model. However, the rise velocity of micro-bubbles appears to be the weakest in suspensions WW1-WW3 and WW5 that mostly contain materials originating from mechanical pulp, e.g. wood based extractives, colloidal pitch, etc. Their appearance is not seen in present analyses of water properties and would thus make ideal continuation for this line of study.

\section{Conclusions}

A digital imaging method was used to present the degree on which apparent viscosity, surface tension and solids consistency affect micro-bubble formation, drag and rise in dilute suspensions. Similarity of micro-bubble formation and uniformity of drag coefficient development in white waters was shown with data needed to devise a novel model describing drag forces affecting micro-bubble rise in white waters. Effects of contaminants in process water on dynamics of micro-bubbles were shown to greatly affect the rate of bubble evacuation and thus the kinetics of suspension deaeration process. Results revealed that contamination decreases bubble size and reduces the micro-bubble concentration. The results can be explained with the changes in surface tension and viscosity of the suspension. In white waters, the drag co-efficient of microbubbles is higher than in pure water when $\operatorname{Re}_{\mathrm{B}}<10$ and lower when $\operatorname{Re}_{\mathrm{B}}>10$.

\section{Acknowledgements}

Authors would like to thank Tekes (The Finnish Funding Agency for Technology and Innovation), PaPSaT graduate school and Academy of Finland for financially supporting this research.

\section{References}

[1] Stoor, T. Air in pulp and papermaking processes, Oulu Univ. press, pp. 66, 2006.

[2] Helle, T-M. Qualitative and quantitative effects of gas content on papermaking. Paper \& Timber, 82(7), pp. 457-463, 2000.

[3] Heindel, T.J. Bubble size in cocurrent fiber slurry. Industrial and Engineering Chemistry Research, 41, pp. 632-641, 2002.

[4] Reese, J., Jiang, P. \& Fan, L-S. Bubble characteristics in three-phase systems used for pulp and paper processing. Chemical Engineering Science, 51(10), pp. 2501-2510, 1996. 
[5] Margaritis, A., te Bokkel, D.W. \& Karamanev, D.G. Bubble rise velocities and drag coefficients in non-Newtonian polysaccharide solutions. Biotechnology and Bioengineering, 64(3), pp. 257-266, 1999.

[6] Okazaki, S. The Velocity of Air Bubble Ascending in Aqueous Solution of Surface Active Substance and Inorganic Electrolyte. Colloid \& Polymer Science, 185, pp. 154-157, 1962.

[7] Hubbe, M.A. Water and papermaking 2, white water components. Paper Technology, 48(2), pp. 31-40, 2007.

[8] Garver, T.M., Xie, T.B. \& Kenneth H. Variation of white water composition in a TMP and DIP newsprint paper machine. Tappi Journal, 80(8), pp.163-173, 1997.

[9] Lindken, R. \& Merzkirch, W. A novel PIV technique for measurements in multiphase flows and its application to two-phase bubbly flows. Experiments in Fluids, 33, pp. 814-825, 2002.

[10] Honkanen, M., Saarenrinne, P., Stoor, T. \& Niinimäki, J. Recognition of highly overlapping ellipse-like bubble images. Measurement Science and Technology, 16, pp. 1760-1770, 2005.

[11] Honkanen, M. \& Marjanen, K. Analysis of the overlapping images of irregularly-shaped particles, bubbles and droplets. Proc. of Int. Conf. on Multiphase Flow, Leibzig, Germany, paper 559, 2007.

[12] Honkanen, M. Direct optical measurement of fluid dynamics and dispersed phase morphology in multiphase flows. Univ. print, Tampere, pp. 80, 2006.

[13] Honkanen, M., Eloranta, H. \& Saarenrinne, P. Submersible, planar shadow image velocimetry system for online, in-situ analysis of multiphase flows in industrial processes. Proc. of the $13^{\text {th }}$ Int. Symposium on Flow Visualization, Nice, France, paper 222, 2008.

[14] Kamgar-Parsi, B. \& Netanyahu, N.S. A nonparametric method for fitting a straight line to a noisy image, IEEE Transactions on Pattern Analysis and Machine Intelligence, 11(9), pp. 998-1001, 1989.

[15] Ouellette, N.T., Xu, H. \& Bodenschatz, E. A quantitative study of threedimensional Lagrangian particle tracking algorithms. Experiments in Fluids, 40, pp. 301-313, 2006.

[16] Frank, X., Li, H. \& Funfschilling, D. An analytical approach to the rise velocity of periodic bubble trains in non-Newtonian fluids. European Physics Journal E, 16, pp. 29-35, 2005.

[17] Hadamard, J. Mouvement permanent lent d'une sphère liquide et visqueuse dans un liquide visqueux. Comptes Rendus Mathématique. Académie des Sciences, Paris, 152, pp. 1735-1738, 1911.

[18] Moore, D.W. The boundary layer on a spherical gas bubble. Journal of Fluid Mechanics, 16, pp. 161-176, 1963.

[19] Mei, R., Klaussner, J.F. \& Lawrence, C.J. A note on the history force on a spherical bubble at finite Reynolds number. Physics of Fluids, 6, pp. 418420, 1994.

[20] Haapala, A., Stoor, T., Liimatainen, H., Nelo, M. \& Niinimäki, J. Passive white water deaeration efficiency in open channel flow. $62^{\text {nd }}$ Appita Ann. Conf., Rotorua, NZ, 2008. 